\title{
To fast or not to fast? Metabolic preparation for elective surgery
}

\author{
Olle Ljungqvist
}

Centre for Gastrointestinal Disease, Ersta Hospital, and Department of Surgery, Centre for Surgical Sciences, Karolinska University Hospital, Huddinge, Stockholm, Sweden

\section{Abstract}

Fasting overnight has been the routine before elective surgery for the past century. Fasting guidelines in many countries have recently been modernized and it is now recommended that most patients drink clear fluids up until 2-3 h before anaesthesia. Several studies have shown that if the overnight fasted state is changed into a carbohydrate fed state before the onset of the operation, several further benefits can be gained. In particular, patients treated with carbohydrates at sufficient concentrations $(12.5 \%)$ in a specially designed drink for preoperative use have less preoperative discomfort, and fewer metabolic derangements postoperatively. Of special interest is the finding that this treatment (or a 30\% glucose infusion given at a fairly high rate) results in substantially less postoperative insulin resistance. This is important, since insulin resistance is the underlying mechanism behind hyperglycaemia, which has been shown to be detrimental in postoperative patients. Recent studies show that preoperative carbohydrates counteract the key disturbances that cause hyperglycaemia, primarily increased endogenous glucose production and reduced glucose uptake in peripheral tissues. This paper reviews the concept of preoperative carbohydrates as opposed to overnight fasting, the mechanisms of action and clinical outcomes for this treatment.

Keywords: elective surgery; glucose metabolism; insulin resistance; preoperative fasting

\section{Background}

Preoperative fasting was first suggested only 2 years after the introduction of general anaesthesia in 1848. The suggestion resulted from the first reported anaesthesia death that occurred in Edinburgh, when a young woman died from aspiration. She had been anaesthetized using chloroform shortly after consuming a meal (1). The pioneers of anaesthesia realized that if the stomach was not empty at the time of anaesthesia there was a risk of vomiting and aspiration. Hence the suggestion arose that an empty stomach should be ensured at induction by allowing sufficient time for it to empty. The dogma of overnight fasting was further supported by studies in the 1940s and has been the reigning routine until recent years.

In the late 1980s paediatric anaesthesiologists were the first to question the overnight fasting routine (2). They showed that it was not only safe but also easier to anaesthetize children if they were allowed to drink before anaesthesia. These first studies were followed by a number of others in large patient groups covering the spectra of surgery, which showed that it was perfectly safe to allow patients to drink clear fluids up until $2 \mathrm{~h}$ before elective surgery. Based on these findings, many countries have changed their routines, and recommendations from an increasing number of national anaesthetic societies now recommend intake of clear fluids (water, coffee, tea without milk and some juices) up until 2-3 h before surgery (for a review see Ref. 3). The driving force behind this change has been that drinking reduces the discomfort of thirst during the preoperative hours.

Another aspect of the overnight fast has recently been highlighted, namely the metabolic effects of being operated in an overnight fasted state. Although this certainly has been the practice and hence the normal situation for over a century, being in the fasted state may not necessarily be the optimal metabolic state in which to tackle the stress of surgery. The overnight fasted state would for most people be a very odd way to prepare for any other kind of stress, and hence could be considered suboptimal in surgery as well. Animal and human studies in the author's laboratory have supported this hypothesis, as summarized before in this journal (4), and will be further reviewed below. 


\section{Minimizing stress and improving outcomes in surgery}

A great number of improvements has been made over the years in surgical practice. Most of the key advances both in anaesthesia and surgery have one feature in common: they reduce the stress for the patient in the perioperative period. It has also become evident that the response to an injury, such as surgery, is the result of complex series of reactions involving endocrine and inflammatory systems. These reactions play a part in the development of many of the metabolic changes occurring after surgery, and also in the development of postoperative complications. To complicate matters further, the endocrine and inflammatory response can interact, and both systems are further activated upon a second wave of stress in the case of complications. An overall schematic picture of the interrelations between these systems and events is given in Fig. 1.

Many of the treatments introduced in perioperative care act by reducing or blocking the activation of the inflammatory reactions, such as minimally invasive surgery, corticosteroids or non-steroidal anti-inflammatory drugs. Others act by affecting the stress hormones. Epidural anaesthesia in the lower thoracic levels blocks the catecholamine response to surgery (5). The overall effect of these treatments is an attenuation of stress responses and catabolism. Furthermore, it seems that when the treatment is given before the onset of the stress, i.e. before surgery, the effect of these treatments is further enhanced. For instance, if epidural analgesia is activated after the operation, the blocking effect on the adrenals to minimize catecholamines in response to surgery is lost, although pain may be

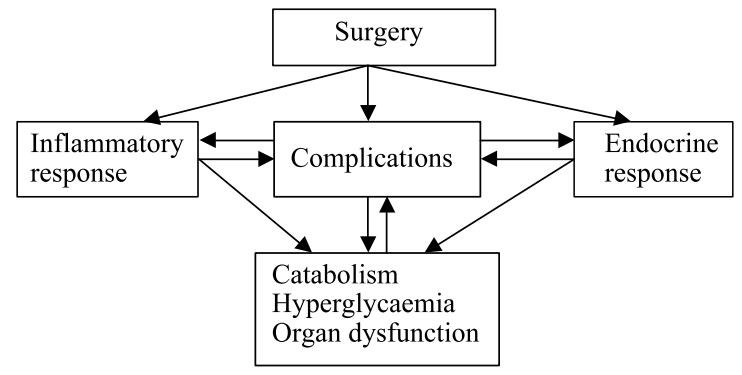

Fig. 1. Overview of the interplay between surgery, the inflammatory response, endocrine reactions, complications and metabolism. Surgery induces both inflammatory and endocrine reactions. These will interfere with metabolism so that a state of catabolism is instituted. A catabolic metabolism may cause complications, which in turn will enhance both the inflammatory and endocrine reactions to enhance the catabolic state further. effectively treated. Thus, starting the treatment once surgery has begun results in an unnecessary activation of stress responses and catabolism.

\section{Metabolic preparation before surgery}

A similar reasoning to that for preoperative activation of epidural blockade of catecholamine release is likely to be behind the efficacy of preparing the metabolism of the patient before the onset of surgery. The natural diurnal pattern of metabolism in humans involves a daytime metabolism dominated by intermittent intake of food and release of insulin (Table 1). Since the effects of insulin last for about $4-5 \mathrm{~h}$ after food intake, the body remains in a substrate-storing state from the first intake of food, usually breakfast in the morning, until it is time to sleep in the evening. It is only during the later phase of the night that body metabolism changes into a fasting state, releasing stored substrates. Thus, before breakfast, an overnight fasted state is in place. At this time insulin is low, and the body is consuming energy stores and preparing for a day potentially without food. Once breakfast is taken in, the body changes back to daytime metabolism. From this basic summary of metabolic regulation it is evident that the natural metabolic state for humans during daytime is a fed state rather than an overnight fasted state. The overnight fast before surgery should, from a metabolic point of view, probably best be regarded as an unnatural state to prepare the body for stress. Hence, the hypothesis behind the questioning of this traditional overnight fasting routine for surgery was simply that it would be better for the body to prepare metabolism in a normal way rather than entering the stress of

Table I. Simplified overview of diurnal changes in endocrine release that govern body metabolism

\begin{tabular}{lll}
\hline & Day & Night \\
\hline Hormones & Insulin + & $\begin{array}{l}\text { Insulin }- \\
\text { Glucagon }+ \\
\end{array}$ \\
& & Glucocorticosteroids + \\
Substrates & Storage & Breakdown \\
Utilization & Carbohydrates & Fat \\
\hline
\end{tabular}

The effects of insulin remain for about $4 \mathrm{~h}$ after each meal. The fasted state is only present for a few hours during the night and early morning until the first meal is consumed. The table shows the principal difference in metabolism in patients operated in the overnight fasted state (Night) compared with patients operated after treatment with carbohydrates shortly before the operation (Day). 
surgery in a state that in all other situations of stress would be unnatural.

A series of animal studies of severe stress clearly indicated that the change in metabolic setting before the onset of stress was disadvantageous (4). The animal data suggested that carbohydrate metabolism at the onset of surgical trauma was an important determinant of the magnitude of the metabolic stress reactions. The data also suggested that manipulation of metabolism using simple carbohydrate intake would result in a different reaction if given before the onset of the surgical stress rather than after the stress had begun.

\section{Insulin and the stress hormones}

Under normal unstressed conditions, a carbohydrate load elicits a prompt release of insulin, which in turn activates a series of metabolic reactions. These include a swift reduction in endogenous glucose release, activation of glucose transporting systems in insulin-sensitive tissues and activation of enzymes securing storing of excess glucose as glycogen. If, however, the same load of carbohydrates is given once a stress reaction is in place, the metabolic effects of insulin are all hampered by the influence of catecholamines, glucocorticosteroids, glucagon and growth hormone, all of which oppose the action of insulin. This forms the basis for stressinduced insulin resistance. What is less known, and only recently discovered, is that insulin may modulate the role of the stress hormones as well, and in this way modulate the endocrine stress response to surgery. Recent studies show that if the patient is operated on under the influence of insulin while given a fairly high load of intravenous glucose (as opposed to the overnight fasted state with no insulin governing metabolism), glucagon release is attenuated and cortisol release is completely abolished (6). In addition, preoperative carbohydrate loading, causing a release of insulin, will result in higher levels of free insulin-like growth factor-1 (7). Hence, there are three key features that from an endocrine point of view are changed by preoperative carbohydrate loading. These help to explain why metabolism is far less catabolic in the postoperative situation in patients given preoperative carbohydrates instead of undergoing an overnight fast. But why is it important to maintain a more balanced metabolism after surgery?

\section{Hyperglycaemia and insulin resistance}

Although it has been well known for many years that glucose control in diabetics is the key to avoiding complications after surgery, it was only in 2001 that it was reported to be important in nondiabetics in the postoperative situation (8). Normalizing blood glucose concentrations in postoperative patients with insulin infusion therapy reduced postoperative mortality in patients in need of ventilatory support in the intensive care unit by $43 \%$. The reduction in mortality was due to a halving of septic complications and associated with large reductions in the need for ventilatory support and in organ failures (notably kidney failure and polyneuropathy). This report from over 1500 patients clearly demonstrates that metabolic control is a key integral part of recovery and outcome in surgical stress. Follow-up studies showed that the degree of glycaemic control was an independent predictor of outcome, while the insulin dosage had a lesser role (9). The degree of hyperglycaemia is a simple reflection of the degree of insulin resistance.

Postoperative insulin resistance has been reviewed in detail elsewhere (10-12). In brief, insulin resistance develops during the operation and remains for about 2-3 weeks after a medium-sized open abdominal operation. The magnitude of insulin resistance increases with the size of the operation, intraoperative blood loss and the time of surgery, whereas gender and preoperative insulin sensitivity have no influence. In most studies whole body insulin sensitivity has been studied using the hyperinsulinaemic clamp method $(6,12)$. With the addition of isotopic tracers, indirect calorimetry and biochemical analysis of biopsy material, the key pathophysiological features of postoperative insulin resistance have been described. Thus, endogenous glucose production is increased, glucose uptake by the cells is reduced, and in muscle glucose transport hampered, as is glycogen deposition. All these factors work in the same direction: to increase blood glucose levels.

In addition, closely associated with the changes occurring in glucose metabolism after surgery are the simultaneous well-described changes occurring in protein and fat metabolism. Thus, protein losses and lipolysis are both minimized in postoperative hyperglycaemic patients once glucose levels can be controlled by insulin infusion (13). This demonstrates that, perhaps unsurprisingly, insulin plays a 
role in protein and fat metabolism, not only during normal conditions but also in postoperative stress.

\section{Clinical impact of postoperative hyperglycaemia and insulin resistance}

In diabetic patients, hyperglycaemia has been closely associated with increased postoperative infection rates (14). Poor glucose control affects the microbial defence capacity in several ways, including leucocyte chemotaxis and phagocytotic capacity. Similarly, in diabetic patients undergoing surgery, glucose control was reported to reduce infection rates (15) by improving those parameters (16). Similar benefits were found in a large study of non-diabetic patients undergoing surgery (8). Importantly, all complications were reduced when glucose levels were controlled by the reinforcement of insulin action.

In addition to the immediate reduction of severe and potentially life-threatening complications by controlling insulin action and hyperglycaemia, there are other associated benefits reported from insulin treatment in the postoperative period. These include faster wound healing after burn surgery (17) and improved protein economy $(18,19)$.

\section{Can insulin resistance be avoided?}

Two ways of proactively minimizing postoperative insulin resistance are described in the literature. One, mentioned above, is the blocking of the catecholamine response by local anaesthetics given via an epidural catheter (5). This is reported to reduce postoperative insulin resistance by about $40 \%$. The other way is to prepare the patient metabolically by giving sufficient amounts of carbohydrates shortly $(2-3 \mathrm{~h})$ before the onset of surgery (11). Laparoscopic surgery represents a third way to reduce insulin resistance (11).

Preoperative carbohydrate treatment has been shown to reduce postoperative insulin resistance by at least $50 \%$ if given in high intravenous doses ( $5 \mathrm{mg} \mathrm{kg}^{-1} \mathrm{~min}^{-1}$ as a $20 \%$ glucose solution). To allow oral administration of large amounts of carbohydrates, a carbohydrate-rich drink was developed containing $12.5 \%$ carbohydrates mainly as maltodextrin, with low osmolality to ensure fast gastric emptying. This has been shown to be safe in well over 50000 patients and also to be as effective as intravenous glucose with regard to the metabolic effects. Studies in open upper and lower gastrointestinal surgery as well as in hip replacement have yielded very similar and positive results, indicating that the treatment is effective in a variety of surgical populations (10).

The effects of treatment with preoperative carbohydrates have been noted in the main components of insulin resistance. Hence, patients given preoperative carbohydrates have a lower endogenous glucose production and a better preserved glucose uptake. These two factors both act to counteract stress-induced hyperglycaemia. The blood glucosecontrolling effect of preoperative carbohydrates also works in real life, as indicated in a recent thesis (20) (Table 2). In patients undergoing major colorectal surgery with preoperative carbohydrate treatment and epidural anaesthesia, the magnitude of insulin resistance was insignificant and normoglycaemia was maintained even during complete enteral feeding in the first 3 postoperative days, with blood glucose concentrations averaging $6 \mathrm{mmol}^{-1}$ during feeding. This is substantially lower than the 9.5 mmol $1^{-1}$ reported in postoperative enteral feeding in patients undergoing major gastrointestinal surgery after an overnight fast and without epidural analgesia. The combination of two factors acting to minimize insulin resistance shows that even in major surgery it is possible to avoid insulin resistance, and that it is possible to feed patients completely without the insulin and still avoid the risk of hyperglycaemia. In addition to minimizing

Table 2. Overview of the changes in glucose metabolism following surgery in the overnight fasted state (Fasted) and in patients given preoperative oral carbohydrates ( $\mathrm{CHO}$ ) compared with a basal normal state without surgery (Control)

\begin{tabular}{|c|c|c|c|}
\hline Parameter/treatment & Control: not operated & Fasted before surgery & $\mathrm{CHO}$ before surgery \\
\hline Glucose levels (mmol I ${ }^{-1}$ during enteral feeding) & Normal $(<6.0)$ & Elevated $(>9.5)$ & Normal (6.3) \\
\hline Endogenous glucose production & Normal & Markedly elevated & Slightly elevated \\
\hline Glucose uptake & Normal & Reduced & Near normal \\
\hline Glucose storage & Normal & Reduced & Reduced \\
\hline Glucose oxidation & Normal & Reduced & Normal/elevated \\
\hline
\end{tabular}

Values for blood glucose levels are given for values reported during continuous enteral feeding, as summarized by Soop (20). 
insulin resistance, these patients retained a completely normal nitrogen balance during the first 3 postoperative days.

\section{Clinical effects of preoperative carbohydrate treatment}

In the 1980s and 1990s a series of reports showed that loading the heart with carbohydrates (with or without insulin) reduced cardiac complications after cardiac surgery (4).

Over the past few years a few studies have been published indicating clinical benefits from preparing patients with carbohydrates instead of overnight fasting or placebo. In line with reduced postoperative losses of nitrogen with carbohydrate preparation, a Danish group reported that muscle strength was better retained up to 1 month after colorectal surgery if the patients received preoperative oral carbohydrate treatment (21).

In patients awaiting both minor and major surgery, preoperative discomfort is reduced by preoperative oral carbohydrate treatment, compared with placebo. Hence, hunger, thirst and anxiety are all positively affected during the hours before surgery (22). A Danish group recently reported less preoperative nausea in patients given the treatment over a placebo, but was unable to detect any postoperative benefits in 94 patients after laparoscopic cholecystectomy (23). This was in contrast to some preliminary data indicating less postoperative nausea and vomiting after this type of surgery comparing patients given carbohydrate-rich drinks with patients who fasted overnight (24). The mechanism behind these effects on well-being may be related to central serotonin release caused by carbohydrates and insulin (11).

A retrospective analysis of prospectively collected data shows that patients treated with preoperative carbohydrates were discharged from the hospital approximately $20 \%$ more quickly than those fasted overnight (25), suggesting that the treatment enhances recovery after surgery.

More studies are underway to explore the potential clinical effects of this novel way of preparing patients for surgery. However, at this stage, it can already be concluded that preoperative carbohydrate treatment is a simple and safe treatment, which allows most patients to be fed immediately after surgery without major concerns regarding hyperglycaemia, with improved nitrogen balance and less reduced muscle strength. These factors are likely to support a faster recovery after surgery.

\section{Acknowledgements}

This work was supported by grants from the Swedish Research Council (09101) and funds from the Karolinska Institutet, Stockholm, Sweden.

\section{References}

1. Fatal applications of chloroform. Section on Legal Medicine. Edinburgh Medical and Surgical Journal, 1848; 69.

2. Maltby JR, Sutherland AD, Sale JP, Shaffer EA. Preoperative oral fluids: is a five-hour fast justified prior to elective surgery? Anaesth Analg 1986; 65: 1112-6.

3. Ljungqvist O, Soreide E. Preoperative fasting. Br J Surg 2003; 90: 400-6.

4. Ljungqvist O, Nygren J, Thorell A, Hausel J. Perioperative nutrition therapy - novel developments. Scand J Nutr 2000; 44: 3-7.

5. Uchida I, Asoh T, Shirasaka C, Tsuji H. Effect of epidural analgesia on postoperative insulin resistance as evaluated by insulin clamp technique. Br J Surg 1988; 75: 557-62.

6. Nygren JO, Thorell A, Soop M, Efendic S, Brismar K, Karpe F, et al. Perioperative insulin and glucose infusion maintains normal insulin sensitivity after surgery. Am J Physiol 1998; 275: E140-8.

7. Bang P, Nygren J, Carlsson-Skwirut C, Thorell A, Ljungqvist $\mathrm{O}$. Postoperative induction of insulin-like growth factor binding protein-3 proteolytic activity: relation to insulin and insulin sensitivity. J Clin Endocrinol Metab 1998; 83: 2509-15.

8. Van den Berghe G, Wouters P, Weekers F, Verwaest C, Bruyninckx F, Schetz M, et al. Intensive insulin therapy in the critically ill patients. N Engl J Med 2001; 345: 1359-67.

9. Van den Berghe G, Wouters PJ, Bouillon R, Weekers F, Verwaest C, Shetz M, et al. Outcome benefit of intensive insulin therapy in the critically ill: insulin dose versus glycemic control. Crit Care Med 2003; 31: 359-66.

10. Ljungqvist $\mathrm{O}$, Nygren $\mathrm{J}$, Thorell $\mathrm{A}$. Insulin resistance and elective surgery. Surgery 2000; 128: 757-60.

11. Ljungqvist $O$, Nygren J, Thorell A. Modulation of postoperative insulin resistance, by pre-operative carbohydrate loading. Proc Nutr Soc 2002; 61: 329-36.

12. Thorell A, Nygren J, Ljungqvist O. Insulin resistance: a marker of surgical stress. Curr Opin Clin Nutr Metab Care 1999; 2: 69-78.

13. Brandi LS, Frediani M, Oleggini M, Mosca F, Cerri M, Boni $\mathrm{C}$, et al. Insulin resistance after surgery: normalization by insulin treatment. Clin Sci (Lond) 1990; 79: 443-50.

14. Kahaodiar L, McCowen K, Bistrian B. Perioperative hyperglycemia, infection or risk? Curr Opin Clin Nutr Metab Care 1999; 2: 79-82. 
15. Furnary AP, Zerr KJ, Grunkemeier GL, Starr A. Continuous intravenous insulin infusion reduces the incidence of deep sternal wound infection in diabetic patients after cardiac surgical procedures. Ann Thorac Surg 1999; 67: 352-60; Discussion 360-2.

16. Rassias AJ, Marrin CA, Arruda J, Whalen PK, Beach M, Yaeger MP. Insulin infusion improves neutrophil function in diabetic cardiac surgery patients. Anesth Analg 1999; 88: 1011-6.

17. Pierre EJ, Barrow RE, Hawkins HK, Nguyen TT, Sakurai Y, Desai M, et al. Effects of insulin on wound healing. J Trauma 1998; 44: 342-5.

18. Ferrando AA, Chinkes DL, Wolf SE, Matin S, Herndon DN, Wolfe RR. A submaximal dose of insulin promotes net skeletal muscle protein synthesis in patients with severe burns. Ann Surg 1999; 229: 11-8.

19. Woolfson AM, Heatley RV, Allison SP. Insulin to inhibit protein catabolism after injury. N Engl J Med 1979; 300: 14-7.

20. Soop M. Effects of perioperative nutrition on insulin action in postoperative metabolism. Thesis, Karolinska Institutet, Stockholm, 2003. ISBN 91-7349-529-8.

21. Henriksen MG, Hessov I, Dela F, Vind Hansen $H$, Haraldsted V, Rodt SA. Effects of preoperative oral carbohydrates and peptides on postoperative endocrine response, mobilization, nutrition and muscle function in abdominal surgery. Acta Anaesthesiol Scand 2003; 47: 191-9.

22. Hausel J, Nygren J, Lagerkranser M, Hellström PM, Hammarqvist F, Almström C, et al. A carbohydrate-rich drink reduces preoperative discomfort in elective surgery patients. Anesth Analg 2001; 93: 1344-50.

23. Bisgaard T, Kristiansen VB, Hjortso NC, Jaconsen LS, Rosenberg J, Kehlet $\mathrm{H}$. Randomized clinical trial comparing an oral carbohydrate beverage with placebo before laparoscopic cholecystectomy. Br J Surg 2004; 91 : $151-8$.

24. Hausel J, Nygren J, Almström C. Preoperative oral carbohydrates improves well being after elective colorectal surgery. Clinical Nutrition 1999; 18(Suppl 1): 80.

25. Ljungqvist O, Nygren J, Thorell A, Brodin U, Efendic S. Preoperative nutrition - elective surgery in the fed or the overnight fasted state. Clin Nutr 2001; 20(Suppl 1): 16771.

Olle Ljungqvist, MD, PhD

Centre for Gastrointestinal Disease

Ersta Hospital

PO Box 4622

SE-116 9| Stockholm

Sweden

E-mail: olle.ljungqvist@ersta.se 